\title{
KnowBrain: An Online Social Knowledge Repository for Informal Workplace Learning
}

\author{
Sebastian Dennerlein ${ }^{1}$, Dieter Theiler $^{2}$, Peter Marton ${ }^{2}$, Patricia Santos \\ Rodriguez ${ }^{3}$, John Cook $^{3}$, Stefanie Lindstaedt ${ }^{2}$, and Elisabeth Lex ${ }^{1}$ \\ 1 Knowledge Technologies Institute, Graz University of Technology, Graz \\ \{elisabeth.lex, sdennerlein, dtheiler\}@tugraz.at \\ ${ }^{2}$ Know-Center GmbH, Austria \{pmarton, slind\}@know-center .at \\ 3 University of the West of England, UK \{Patricia.Santosrodriguez, \\ John2. Cook\}@uwe. ac.uk
}

\begin{abstract}
We present KnowBrain (KB), an open source Dropbox-like knowledge repository with social features for informal workplace learning. KB enables users (i) to share and collaboratively structure knowledge, (ii) to access knowledge via sophisticated content- and metadatabased search and recommendation, and (iii) to discuss artefacts by means of multimedia-enriched Q\&A. As such, KB can support, integrate and foster various collaborative learning processes related to daily work-tasks.
\end{abstract}

Keywords: collaborative learning, meaning making, informal learning

\section{Introduction}

Today's challenges in industry and research require workers and learners to effectively and efficiently communicate and collaborate within different disciplines in the digital world. Hence, tools are needed that facilitate social and collaborative learning or working in informal settings by supporting underlying meaning making processes [8]. KnowBrain (KB) aims to do just that. It is a social Dropbox-like system representing an online knowledge repository enriched with social features beyond sharing and organizing mechanisms: e.g., multimedia question-answer (Q\&A) functionality, activity stream, grouping or social recommenders. Since $\mathrm{KB}$ is self-hosted, i.e., it can be hosted on the own servers within an enterprise, data privacy and control over the system is ensured. Together, those features aim for unfolding the potential of meaning making to enable collaboration in an online social knowledge repository for organizational learning contexts.

\section{Background}

Informal workplace learning takes place alongside the daily work-tasks on a justin-time basis. It is problem based, contextualized, episodic and includes the communication and transfer of knowledge and experiences [1/344. Hence, informal learning experiences often involve other persons and lead to a social context [6]. 


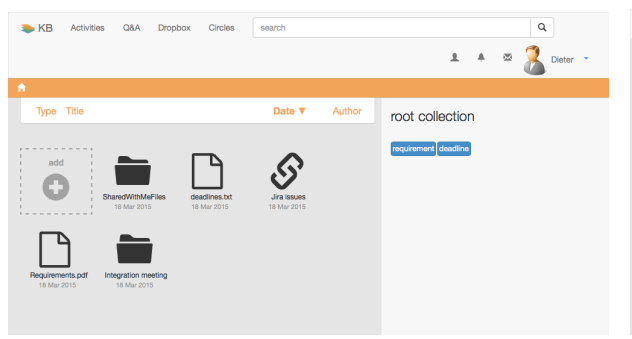

(a) Dropbox-like online knowledge repository

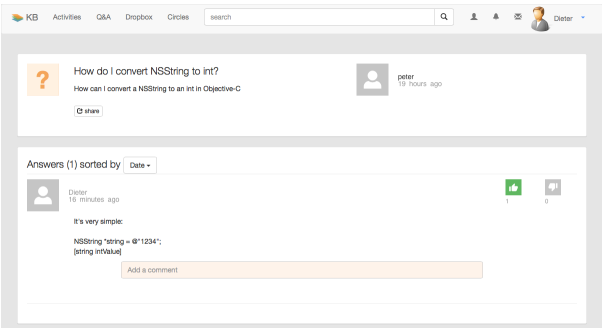

(b) Answered question in Q\&A

Fig. 1. KnowBrain's knowledge repository and question-answer (Q\&A)

In other words, informal workplace learning reflects complex situations with many interwoven actors and artefacts (e.g., documents, links, pictures). This situation requires that we repeatedly establish a shared understanding to be able to collaboratively solve problems and arrive at an agreed solution. This can be achieved through meaning making. It takes place when multiple collaborators contribute to a composition of interrelated manipulations of digital artefacts 9. Hence, meaning making processes have to be considered, supported, reflected and exploited as part of social and collaborative learning tools 2. KB builds the ground for such meaning making processes by enabling manifold ways of sharing and collaboratively manipulating artefacts, which are a necessary prerequisite for collaborative and organisational learning [7]. In addition, KB makes use of respective outcomes, the metadata, through recommendations such as collaborative filtering to further stimulate meaning making.

\section{KnowBrain: Prototype Description}

KB provides an integrated solution to (collaboratively) collect and structure knowledge, to reuse and share information within a single platform, to access information via search and intelligent recommendation mechanisms and to enable multimedia-enriched Q\&A, discussions and chats. It opts for openness and loosely coupling of functionality by decoupling client-side business (i.e., solely relying on HTML, CSS and Javascript backed-up by the AngularJS framework) from server-side REST-based infrastructure (i.e., the SocialSemanticServer - SSS - a service-framework for social semantic network data (5) in comparison to monolithic and closed systems as they can be found in (organisational) knowledge repositories (e.g., Moodle or Blackboard from within the e-learning scene, or Sharepoint from within the enterprise content management area). Thus, KB eases its extension with new and adaptated existing affordances needed for informal learning. It aims to integrate popular approaches from within otherwise separated environments such as content management platforms (e.g., Dropbox or Google Drive), social platforms (e.g., Facebook or Google+) and help seeking 


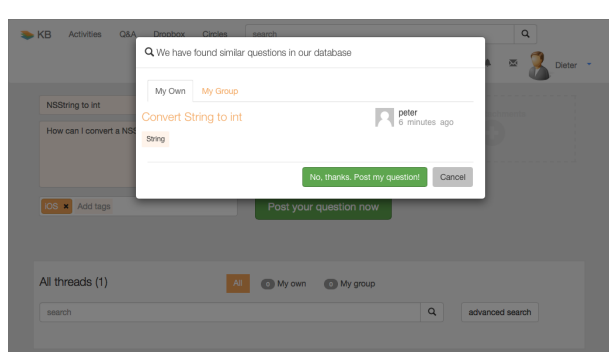

(a) Recommendations in Q\&A

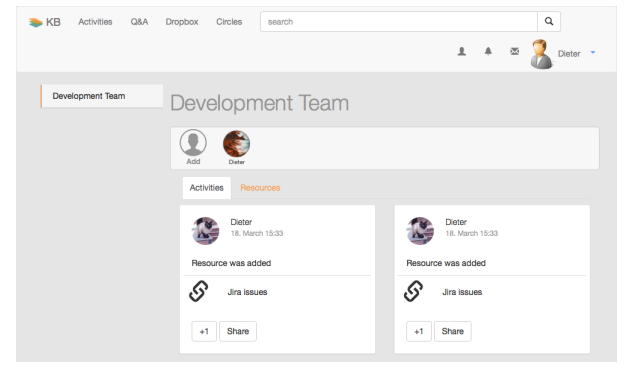

(b) Activities within a grouping

Fig. 2. Example for KnowBrain's recommendations and activity stream

platforms (e.g., StackExchange) with research findings in the area of recommendation, search and meaning making. Figure 1 and 2 show the current status of the prototype which enables sharing, social tagging and collaboratively organizing of (uploaded) artefacts such as learning or working material, in addition to hierarchically structuring the same in folders (see Figure 1 1 ). Thereby, sharing can be done directly with colleagues, friends, groups or the whole community. Collaboratively enriching artefacts with more or less formal concepts (i.e., tags or categories) is inherent to meaning making driven by technology. Hence, KB also supports learners in their needs to discuss artefacts or to raise questions with regard to particular topics of interest as shown by Figure 1 $1 \mathrm{~b}$. Interaction threads in KB can not only be enriched with comments and metadata, but also with multimedia artefacts from the local file system, or from already organized content in the platform. To scaffold learners with the task at hand, KB recommends similar threads or answers before new communication threads are created (see Figure 2a). These threads can in turn be shared with others to make them visible to a broader community. To allow KB users to keep track of meaning making outcomes and their evolution, activities of collaborators can be followed via user- and group-based profiles as shown in Figure $2 \mathrm{~b}$. These profiles also provide insights into the interdependency of artefacts, users, metadata, activities and communication threads. This way, users can better understand their learning or working groups and acquire relevant knowledge to further minimize interruptions of the ongoing meaning making process. Search can be utilized to find (multimedia) artefacts, users, groups and metadata using metadata and content-based keywords. On top of this, KB offers recommendations to enrich search results with interesting and relevant learning materials. Thus, the emerged traces of meaning making are reused and exploited to scaffold community members.

\section{Conclusion and Future Work}

The current prototype of KB leverages essential meaning making processes via collaborative (re)structuring, discussing and enriching of learning materials to 
enable online collaboration. In the future, we will integrate further intelligent services that take into account research in technology enhanced learning from within various areas such as sense making or expert and community identification. Planned features include graphical interaction mechanisms facilitating social categorization processes, as well as opportunities for collaborative writing to formalize and mature ideas also in other formats. All these efforts aim for a seamless connection of workers and learners via thoughtfully integrated features in a self-hosted knowledge repository that shall also effect real-life working processes in the long run.

\section{Acknowledgements}

This work is funded by the Know-Center and Learning Layers. Learning Layers is funded by the European Commission within the 7th Framework Programme (Grant Agreement 318209). The Know-Center is funded by the Austrian COMET Program under the auspices of the Austrian Ministry of Transport, Innovation and Technology, the Austrian Ministry of Economics and Labor and by the State of Styria. COMET is managed by the Austrian Research Promotion Agency FFG. We thank Viktoria Pammer-Schindler for her valuable feedback on the paper and Christoph Trattner for his work in the context of Learning Layers in the first 1.5 years of the project.

\section{References}

1. Attwell, G., Baumgartl, B.: Creating learning spaces: training and professional development for trainers. Vienna, Austria: Navreme (2008).

2. Dennerlein, S.: Understanding and Supporting Intersubjective Meaning Making in Socio-Technical Systems. EC-TEL Doctoral Consortium (2013).

3. Eraut, M.: Non-formal learning and tacit knowledge in professional work. The British Journal of Educational Psychology, 70(1), 113-136 (2000).

4. Kooken, J., Ley, T., De Hoog, R.: How do people learn at the workplace? Investigating fourworkplace learning assumptions. E. Duval, R. Klamma \& M. Wolpers (Eds), Creating new learning experiences on a global scale, 158-171. Heidelberg, Springer (2007).

5. Kowald, D., Dennerlein, S., Theiler, D., Walk, S., Trattner, C.: The Social Semantic Server A Framework to Provide Services on Social Semantic Network Data. In Proceedings of I-Semantics (2013).

6. Marsick, V. J., Volpe, M.: The Nature and Need for Informal Learning. Advances in Developing Human Resources, 1(3), 1-9 (1999).

7. Pammer, V., Prilla, M., Divitini, M.: The Functions of Sharing Experiences, Observations and Insights for Reflective Learning at Work. Proceedings of the 2nd Workshop on Awareness and Reflection in Technology-Enhanced Learning (ARETL 2012) at EC-TEL 2012 (2012).

8. Stahl, G., Koschmann, T., Suthers, D.: Computer-supported collaborative learning: An historical perspective. Computer, 409-426 (2011).

9. Suthers, D. D.: Technology affordances for intersubjective meaning making: A research agenda for CSCL. International Journal of Computer-Supported Collaborative Learning, 1(3), 315-337 (2006). 